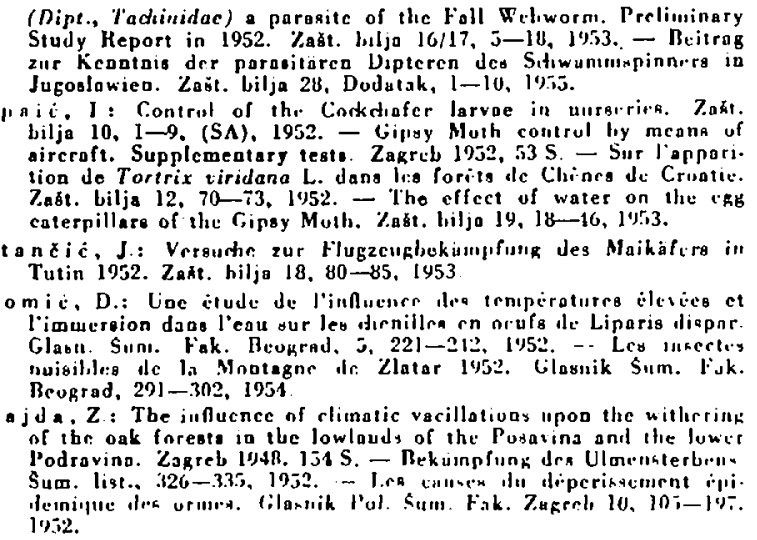

(Dipt., Padiuidac) a parasite of the Fall Fellworm. Prelioninary

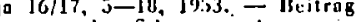
Jugoslawien. Zaŭt. Lilja 28, Dodatak, 1-10, 19.5.

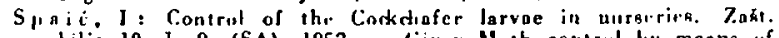
bilja 10, 1-9. (SA), 1952. - Giliyy Muth cotitrul liy meana of aircroft. Supplementary teste. Zakrib 1052, $53 \mathrm{~S}$. - Sur l'oppori. tion do Tortrix riridana L. dana lia forits de Clicars de Cruntie. caterpillare of the Gipsy Muth. Zast. hiljo 19, 18-16, 1'jis.

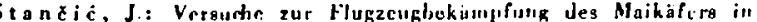
Tutin 1952, Zakt. hilja 18,80-85, 1953

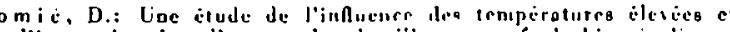
limurgion dans leau dur ley dirnillen on orufs de Liparis iliapnr

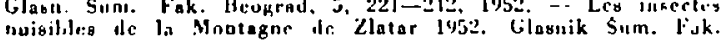
Beograd, 291-302, 1951

j $d a, Z$ : The infuence of climatic vacillations upon the witlerint

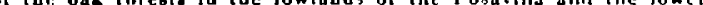

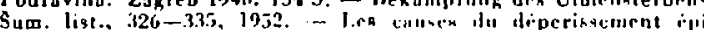
livi2.

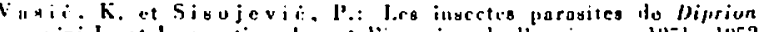
ini I.. et leur

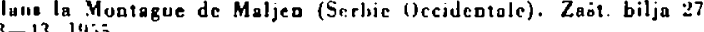

irnik. F: Die Fiditenblattwespe und unyere Erfolirungen ill ihrer Bckumpfung. Goderski Vestuik 9, 20y-2:20, 1\%:1.

Zecevic, J): Winter facding nud dinclopnint of the Gipsy Moth

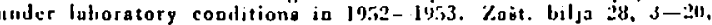
1955.

7ivajillovie, S.: Dic Cradation der Burklukufer in len Naldel. nuld.ru Serbiens 19.15/47. Godn. Pol. Sum. Fuk. Hengral, 65-7. (SA. ". J.). - I.es Scolytes de la moutazne Guliju. Clannik Sulı. Jink. 1, 209-310, 1950- Role of aireruft in Gipsy Minth control

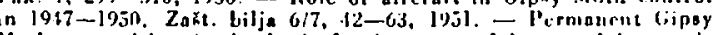
Anth supervision is the lanis for its succeasful control in woods 4 nd ordiords. Zabt. bilja 10. 1-4 (SA). 1953. - The Gipey Moll cluatera in Eaat nod Soutb.Eogt Srrbin in 19:3. Zabt. bilja 211.

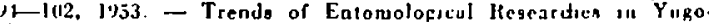

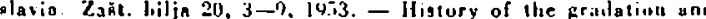

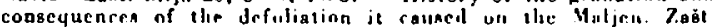
liiljn 2t, 1-19, 19jt. - A Centriliution to the hnowledge of Sculgtidue ou the Maljen Mouninin Flaan. Sunı. Fak. 8, 3-2") 195

Aus der Biologischen Bundesanstalt für Land-und Forvtmirtstha/t, Insfitut für biologisshe Schödlingsbrliämpfung und Kurtoffelliäfer-Forsthung. Durmstadt

\title{
Die gegenwärtige Situation der biologischen Schädlingsbekämpfung in Deutschland
}

\author{
Voll J. Fran\%.
}

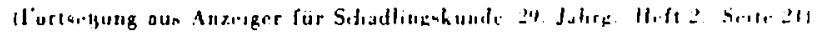

III. Arbeiten des Institutes für hiologische Solü̈llingshe.kïmpfung der BIBA. Jurmatudt

Wie ohen hercits allgedellet. oullen all diescen mil 5) Wissensctuftlern hesebten Spezialinstitut vor allem soletie Forschungsanfgabeu bearlicitet werden, die gloids. zeitif cincn erhöhten technischen Aufwand und das 7.u. saummenarleciten mehrerer Spezialisten crlordern. Miersu gehören vor allem:

1. Die Verwendung von Mikroorgunismen aur hiologisctenn Rekämpfung von Stiadinscktcn. 2. Dic Einfuhr und Masirumutt von Fntonopliagen zur liekämpfung pinzeschleppter Pflanzenfeind.: und Unkräuter. Da dem InsliIIIt ein Instektarium nod fehlt, konnte hisher nur das crste Thema bearbeitel werilen. Daturhen find Giundlagenarbeiten üloer dic Wirkungsweixe natürlicter Foinde augelaufen. Lurr Erfüllunir dienter Aufgalinn ist das Insti. tut in Irei Sektionen geqlicdert. Dic Mitarbeiter der Sch. lion, "Okologiı:" verfolgen im Freiland den Massenwertiocl liestimmter Sotladiusckien. unter besonderer Berücksidhligung der biotistuen Begrenzungefaktoren. Dabei wird unter Einschaltung erginzender Jahoraloriumsexpori.

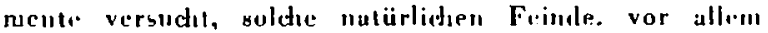
Krankheitserreger, an finden, die sid, zum Finsab durd, Jen Menstien rignen; ihre Bedcultullg in Gradaliens. ablunf wird dalepi mersend verfolgt. In der Srktiun ,Iusckleupallonlogie" werden die selbst gesanmellen oder von anderen Stellen ringesandten kranken haw. toten Insckten untirsuctl. Diagunse. Isolicrung und


kmuzeirhne"l die weitere Bearlecilung. Liegen vielurr. nprefiende Kranklecitscresuer sor, so verden eic in Laboratoriums. und später in Freilandsersuchen crprubt. in Zusammenarbeit mit Jer Sektion ..Okolngie" und versthiedenc'n underen Institulen. Wie Sektion "Histopialhologie" trägt srhlicblich durch histologisdue Untersuctunnzen gesumder und kranker Insekten dazu hei, den Vorgatug und dic Wirkung der Infektion im Wirtsortanismus verstehen und diagnoslizicren 7.u kölnen. Stetigar Cedanken-

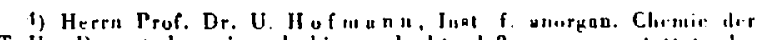

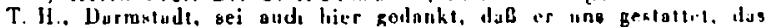
lilektrosummikroskon suines Institutey mit an lirnisgen. austausct mutereinander und Konsutration aller Mit. arbriter auf gewisse ..Instituteprojekte" haluen sids gut bewïhrt. In folgender wird üleer ejerige latefende und ah-

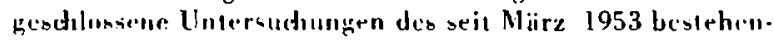
den und heil April 1951 persuncll voll beboblen Inetilutes stictiwortartig berichtet.

1. Nalelholeblattweopen. Anfangs wurden die maliirlictien Sterblichkeitsfaktoren der in Kirfern. jugend.on schüdlictien Roten kicfernbusctihornblatlwespo (Nerodiprion sertifer |(icoffr.]) sludicrt (19). Der Firreper einer dubei gefundenen Viruse wurle dargestallt. die Grumdligen unter besonderer Berürksithligunte des Prohiloms latentar Virosen mutersuctit (33). Dip Seludie licks sich beci äuburlidı gesunden Tieren durch Behatudlung des Futtera mit Thioglycolsäurc, Natriumfluorid oder anderen Stoffen. außerienm ilurch UV'-Bestrahlung. provoxicren (38).

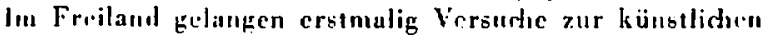
Infeklinn endemibrtier Jlattwespon mil einer ebenfalls endemisdu('n Virusseudie (23). Ferner telang dor Nach. wris, daß die Polyederviren der genannte" Art nach einer Passage durch den Darm einer Raulowallec (Rhinocoris immolatus I.) otler cincs Rotkchldhens (Erilhucus rubrrula I..) infektiös lleilien. Damit Hewinnen Priidutur("n Heringer direkler Wirkung cine crhöhte Bedlutung "l, Vektoren von Polyedersendien (24). - Dic Krankhuiten anderer Haltwespen werden in Zusammenarbcit mit der Nipilersüitıs. Furstl. Versllchsanstalt. Aht. B, Götlingen. studiert.

2. Fugerlingevon Mrlolontha. Durdinudulen von rexelmäßize inn Freiland gesanmelten Engerlingen rerlauhten es, den Cresumdheilszustand der Population und dic perpulationsdynamiorhe Wirkung von versshiedrnen Scudee" grnauce uls hisher möglich жu beurteilen (4B). Parallel Jaufen Arlueiten üleer die Ferreger der Fingerlingsseurticu, muler lenen eine Ricketlsiuse besonders withlige int $(34,47)$ und eine Microsporidiose neu besclirichen wisd (3.). Lum frühacitigrn Nachweis der Rirkettsien wird nenerelings die Serodiagnose herangezogenl (3i).

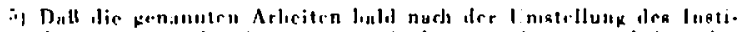

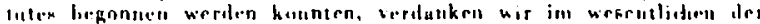




Infektionsversuche mit einheimischen und eingeführten Erregergruppen schaffen die Grundlagen für die zu einem späteren Zeitpunkt geplanten Arbeiten im Freiland.

3. Tannentriebwickler (Cacoecia murinana $\mathrm{H}$ b.). Untersuchungel über die Bedeutung biotischer Mortalitätsfaktoren aus dem Randgebiet einer Masscnvermehrung im Sdhwarzwald (zusammen mit der Vogelschubwarte Ludwigsburg, der die vogelkundlichen Arbeiten obliegen). Eine Kapsel-Virose (Granulose) hat im Zentrum der Kalamität andere Bedeutung als im Randgebiet, eine Polyeder-Virose wird erstmalig nachgewiesen. Die Ergebnisse dieser Arbeiten sollen der forstlichen Praxis Bekümpfungsmethoden an die Hand geben, die entweder unter Verwendung oder bei weitgehender Schonung von Nuţorganismen die Gesamtbiozönose möglichst wenig verändern.

4. TannenI ä use. Bei der Tannenstammlaus (Adelges piceae [Rats.]) ließ sich durch Anwendung neuartiger Kartierungsmethoden und Freilandexperimente erstmalig bei einem nur von Raubern (nicht von Parasiten) in Schach gehaltenen Insekt feststellen, wann und wie stark der Einfluß natürlicher Feinde im Verglcich zur Veränderungr des Nahrungssubstrales wirkt $(17,22,30)$. Diese Arbeiten sind die Grundlage für das Verständnis des Massenwechsels anderer, z. T. schädlidherer Tanncnläuse; dadurd ergeben sich neue Ansaţpunkte für Bckämpfungrsmaknahmen.

5. Baumweißling (Aporia crataegi) ${ }^{6}$ ) und Wiesenschnake (Tipula paludosa $)^{7}$ ) zeigen interessante Viruskrankheiten, bei denen zu klären ist, wie sie den natürlichen Gradationsablauf beeinfussen und ob sie für künstliche Infektionen und damit zur biologischen Bekämpfung im Freiland geeignet sind, - Weitere derartige, zusammen mit anderen Instituten geplante Arbeiten sind im Anlaufen.

6. Allgemeine Insektenpathologie. Uber durchschlagende Erfolge hei der Bekämpfung mancher Schadorganismen durdh künstliche Verbreitung spezifisder Krankheitserreger liegen aus dem Ausland neuere Beispiele vor (20). Vor allem soldhe Seuchen scheinen sich für eine praktische Verwendung zu eignen, dic - einmal ausgebracht - den Bevölkerungsstand ihres Wirtes stetig senken können, z. T. durch dic Hilfe spezifischer Úberträger $(21,69)$. Dazu kommen Epizootien, die nur deshalb selten lange andauern, weil ihre Ausbreitungsfähigkeit zu gering ist; hier kann der Mensch gelegentlich durch künstliche Verbreitung nachhelfen. - Bei der Inangriffnahme eines newen Forshungsgebietes ist es notwendig, allgemeine Methoden und Grundlagenfragen mit zu bearbeiten, damit später die auf praktische Anwendung zielende Arbeit darauf aufhauen kann. Einsendungen kranker Insekten erfordern laufende Diagnosearbeiten, um solche Virosen, Bakteriosen, Mykosen und Protozoonosen sicherzustellen, die sich für weitere Infektionsversudie eignen. Die Methoden zur Zucht von Insekten in sterilisierbaren Käfigen und zur färberischen Darstellung von Polyedern in histologischen Schnitten mußten verbessert werden $(40,41)$. Von weiteren insektenpathologischen Grundlagenarbeiten seien hier genanut: IntravitalFluoreszens-Mikroskopie zur Untersuchung zellpathologischer Reaktionen; Hämatologic und Inmunologie gesunder und kranker Insekten im Laufe ihrer Entwicklung; Fehldiagnosen bei Untersuchungen kranken Materials (36).

\section{Internationale Zusammenarbeit}

Die Verwendung von Lebewesen zur Schädlingsbekämpfung ist ihrer Natur nach übernational. Die Verbreitung von Nutgorganismen richtet sich bekanntlich

6) Bearbeitet zusammen mit Herrn Prof. B I u n ck.

7) Bearbeitet zusammen mit dem Institut $f$. Griblandfragen der BBA lediglich nad äkologischen, nidht nach politischen Grenzen. Daher gehört die Pflege des Kontaktes zu den ausländischen Fachinstituten gleicher Arbeitsrichtung mit zu den ersten Anliegen. Folgende Einzelheiten mögen auch einen weiteren Kreis interessieren:

1. In der Internationalen Kommission für biologische Schädlingsbekämplung (CILB), deren offizielle Anerkennung durch die Bundesrepublik wünschenswert ist, werden solche Fragen bearbeitet, die mehrere Staaten gemeinsam angehen. Hierzu gehört z. B. die Entscheidung über Import von Entomophagen oder Erregern von Inscktenseuchen, die curopäischen, haustierartig gehaltenen Nutjinsekten (Seidenspimer) unter Umständen gefährlich werden könnten. Die Kommission, die organisatorisch der Union Internationale des Sciences Biologiques (UISB) angegliedert ist, koordiniert ferner die gemeinsam interessierenden Vorhaben; hierdurch werden Projekte, die einem Staat allein zu kostspielig würden, entscheidend verbilligt. Derartige Pläne werden audh z. T. von der Food and Agriculture Organization (FAO) und der Europäischen Pflanzensdhutgorganisation (EPPO) mit unterstütyt (Beispiel: Einfuhr von Parasiten zur biologischen Bekämpfung des Weißen Bärenspinners, Hyphantria cunea Drury, aus sciner nordamerikanischen Heimat nach Jugoslawien [15]. Zu den für uns widhtigsten Aufgaben der CILB gehört schließlich die Errichtung eines internationalen Bestimmungsdienstes für Nutginsekten, an deren Finanzierung sich die Mitgliedstaaten gemeinsam beteiligen wollen. Hierdurd hoff $t$ man dem für alle Arbeiten unseres Gebietes gefährlichen Mangel an Spezialisten für Entomophagen etwas abhelíen zu können.

2. Durdl die Aufnahme von $G$ astwissens chaf t lern a s dem AusIand wird der persönliche Erfahrungsaustausch eingeleitet. Ein kanadischer Kollege von der Division of Entomology, Canadian Department of Agriculture, konste z. B. vom Darmstädter Institut als „Haupt-

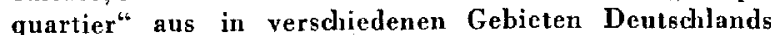
Parasiten des Frostspanners (Cheimatobia brumata L.) sammcln, die zur biologischen Bekämpfung des neuerdings nad Kanada verschleppten Schädlings dorthin verschickt worden sind. Die Besuche führender ausländischer Wissenschaftler gaben wertvolle Anregung für die weitere Forschung. Dem Verfasser wird es durch ein Stipendium der Deutschen Forsdmngsgemeinsdhaft möglich sein, im Sommer 1956 den Einladungen des Science Service des Kanadischen Landwirtschaftsministeriums und verschiedener Institute der University of California Folge zu leisten.

3. Die Gründung der Internationalen Union für angewandte Ornithologie im August 1955 in Frankfurt a. M. wird ebenfalls dazu heitragen, die $\mathrm{Zu}$ sammenarbeit mit den Fachkollegen im Ausland zu fördern. Dadurch, daß ein Deutscher (Dr. Bruns, Würzburg) zum Sekretär dieser Vereinigung gewählt worden ist, ergibt sich für uns die Möglichkeit und Verpflichtung, den verstärkten internationalen Kontakt für die praktische Arbeit zu nutzen.

4. Durdı die frühere Tätigkeit des Verfassers beim Commonwcalth Institute of Biological Control besteht ochließlich noch eine gute Zusammenarbeit und ständiger Erfahrungsaustausd mit dem Europäischen Laboratorium dieser Organisation und seinen Mitarbeitern (Direktor: Prof. L. P. Mesnre).

\section{Ausblick}

Dieser Zwischenbericht über den Stand eines Zweiges der Pflanzenschutforschung, der bei uns noch recht jung ist, verfolgt einen doppelten Zwedk. Einmal soll er all denen, die organisatorisch oder wissenschaftlich an dicsen Fragen mitarbeiten, einen Überblick über das geben, was 
bei uns zur Zeil aul dem Cebict der biologistarn Schäd. lingsbekämpfung treschicht. Um dies zu erreiden, mußte: von der sonst wohl herriünleten Sitte alyewichen werden, mur ühor Arbeitsergelmisge, nidht über Vorhaben zu herithten. Wer die angeführten Publikationen zur Hand nimmt, wird jedodı feststellen, daß sie eine Fülle ncuer und für die weilere Entwieklung der Pflanzenschutzpraxis wichtiger Resultatc bereits anthalten. Der Wer zur l'raxisreife hiologisctier Verfahren ist allerelings, wie schon öfters letont (16), länger als bei underen Verfahren. Zwar zog sich dic Entwicklung der Phosphorsäurcester, um ein I3cispiel zu nennen, übcr Jaliryehnte hin (62). Dann fand man jedoch Mittel, die gleidreitig gregen Dutzonde von Inscktenarten wirken. Ganz anders hei hiologisdon Verfahren: Sie sind zumcist Spexifika segen solche Glieder der Lebensgemeinschaft, dic: -- oft als Folge monschlicher Eingriffe -- bedrohlid überhamd uehmen. Um den je nadı Sdädling und Umweltverhältnissen versdiedenen, jeweils günstigsten Ansą̧punkt zu finden, benötigt die Forschung Zeit. Hier muß jeder Fall für sid hearbeitet werden. Dicsem Nachteil steht gegenuiber, daß die meisten biologisehen Verfahren daucend wirksam bleiben und daß der Sdıädling selbst i. d. R. keine Abwehrreaktionen entwickelt. Aber Zeitvorlust bedentet heute ansdeninend schr viel.

Damit kommen wir zum zweilen Grund dieses Zwi. schenheridites: Er soll ein sachlich fundiertes Gegengewicht bilden gegen all jene Meldungen, die - iber. trieben lobend oder verdammend - dem Wesen biologischer Verfuhren völlig fremd gegenübcrstchen. Jede Schwarz- Weiß-Darstellung, jede Alternativ-Forderung (,Vögel statt Gift" usw.) ist ein Rürkschlag, der zu weitgehenden Mißverständnissen führen kinn (71). Unser Bcmühen ist cs, dann, wenn andere lBekümpfungsmethoden versagen oder beaditliche Nachtcile haben, behutsam hier und dort hisherige Methoden abzuwandeln oder rein biologische Verfahren hereitzustellen, ohne dadurch die nadshaltig gute Qualität der Kulturpflanzen und ihrer Erträge im weiteren Sinne zu gefährden. Dicse Aufgahe ist schwer genug, zumal in einem so intensiv bewirtsctufteten Land wie dem unseren; verlangt sie dod, daß wir aufhören, den schnellen Erfolg zu übersdiitgen, und beginnen, nicht das Tempo der Rotationsmaschinen, sondern den Rhythmus des Lebendigen als das cutscheidende Zeitmaß anzucrkennen. Diese Unstellung wird nur gelingen, wenn weiterhin Staat, Wirtshaft und Praxis diesen Aufyahen aufgeschlossen gegenülerstehen, eingedenk ihrer Verantwortung gegenüber den kommenden Generationen. Sollte es doch das Fernzicl jedes I'lanzensedutges, also aud der biologischen Bekämpfung, sein, das unter unseren Wirtschaftshedingungen erreidhluare Höchstma $B$ an nachhaltiger Regulationsfähigkeit auch in den Kulturlandechaften zu verwirklichen $(42,58)$. Auch das ist eine "Rationalisierung" des Phanzensduntyes, bei dor dic Zukuuft einkalkuliert ist.

\section{Literulurverzeidmis}

(Aua dem gelir umfangreidien Schrifttum sind hier vor allem dic

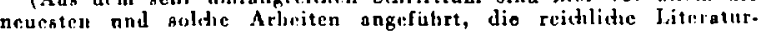
hinweise bringet.)

(1) Babre, F. H.: "Clumical control aud rusistance to insecti-

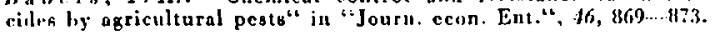
cus.s.

(2) Berker, J.: ,. Mer Einflub zwcirr Raubmillen auf die PopuIntion rai Metacteranychus ulmi Fortrag 31. 1)tadi. Pflanzensiti. Tagung, Kasecl (1955) [im Druck].

(3) Blunck, H.: ..Zur Kenutnia dea Massenwedesels von Pirris brassicae' L. mit lesonderer Berüdksidtigung les Dürrejahres 19.17“" in ,Zeitsdir. \#ngew. Ent.“, 32, 1.11--171, 1950.

(1) Blunck, H.: "Parabiten und Hyperparasiten Yon Pieris relpree L..“" in ,Zfitstir. P'langenkrankh.“,58, 25-54, 1951.

(5) Blunck, H.: ,Mikrogporitlien lu:i Pieris lrassicae L., iluren Parasiten und IIyperparusitu" in : Zcitatir. angew. Ent.", 36, $316-333,1954$
(6) $13 / n \|, k$, II.: ,Beobathungen über Wunderflüge von Pieris brassicue L." in ".,Beitr. Eutom.", 4, 48.-524, 1951.

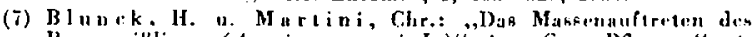

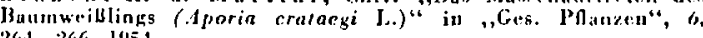
$26.1-266,1454$

(8) B r $n$ ns, H.: ., Breinflussung der Kokondichte ciner Populntion dor Kleinen Fiditinllattwespe (Lyzaconematus abictrm) durth

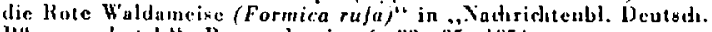

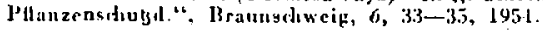

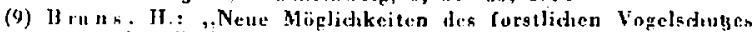

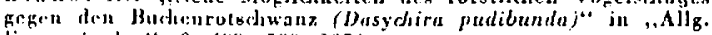

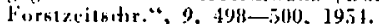

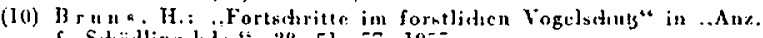
f. Sitailliorskde." $28,51-57,19 \pi, 5$.

(11) Colly er, E. " "K K $\mathrm{r} b y, A$. H. M.: "Sorne factors afferting the bilaner of phytophagens and predacioum mites on apple in

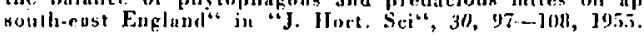

(I2) Cram er, II. H.: ,.Der Stand unserrs Winacns über dic Aluswirhung grobfäidijer Sdiädliugsbekijmpiung auf Waldbiozönosen. Sammolheridt iiber den Stand des Vissens" io ,Zeitsdar. Pflat. zrokrankh.", 62, 422-435, I95.).

(13) Bosse, C.: Aus der Biologie der Roubunilbe Typhlodromus crocumeris Oud. (dear., Phytoscialac)" in ,Zeitutir. P'tlanzen.



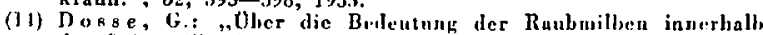

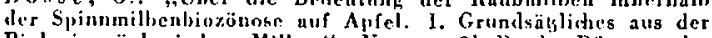
Biolugie räuleristler Milhen", Vortrag 31. Dtsets. Pfanzengdi. Tagung, Kanorl (1955) Jim Druik]

(15) J) rees, H.: "Beitrag zur Kenntuis des Auftrelenr gefiilerlidier

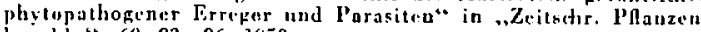
krankh." $60,83-86,79.3 .3$

(16) Franz, J.: "Neue Möglichkeiten nnd Erkebnjobe der lioloki fitien Sibädlingslıckumpfüg" in .,Mitt. Biolog. Zrturalunftalt,

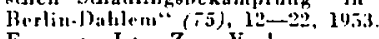

(1i) F r "nz, J.: ,Zum Forkomine"n und Mosenwedisel der Tunden At tummlane Adelges (Dreyfusia) piccae (Ray.) in Nordamerika und Eurupa" il "Verl. Deu. Ges. f. ongew. Ent." (1952), 117-121, list.

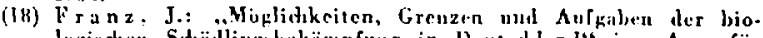

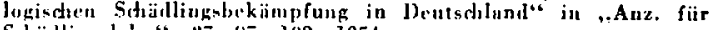

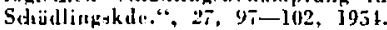

(19) Fran $\%$, J.: .Prolileme nngewander Vogelkunde" in ,J. $f$ Ornith.", 95, 111-123, 195.1.

(20) Franz, J.: Mikrolien kenon Insekten Nene Wege der bio


1955 .

(21) Franz, J.: ,Die kiubstlidus Verbreitung von Virosen einiger Blattwenpen (Diprionidac) inuerhnlls und auberhalh ihres Endemie. gehieter"s" in ,Verl. d. Deutachen Zool. Ges.", Erlangen (19:55) [im 1)rude]

(22) F ran\%, J.: „Natiirlictse Feimle und Nialirpfanze als biozionntiadu: Begrenzumgsaktoren bei Gradationen der Tannenatammlau Adelges (Dreyfusia) piceae", Vortrag 3l. Disd. D'Qunzensdiubtagunk, Kasael (195i) [im Druk].

(23) F ran\%, J. u. Niklas, O. F.: ,Feldversude zur Bekümpfung dur Roten Kiefernlusedilornblattwespo (Neodiprion surtifer [Cicoffr.]) durch künstlictio Verbreitung ciner Virugsendie:" in ..Nartiriditenblatt Deutsd. Pfanzeosdubd." Braunediwcig, o. 131-134, 1954 .

(21) Frang, J., Krieg, A. a. Langenbuch, R.: ,lint:r. suctungen ubrer den FinfuB der Pagenge durdi do:n Darm von Aatuinsekten und Voigeln auf die Infektiosiät inkektenputho. Raubinbeten und Vegeln auf die Intektiokiat inkrktenputho.

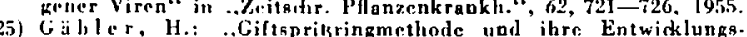

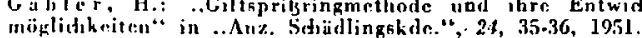

(26) GäBwald K: Dic Rote Waldameise im Dienste der Wall. hysieme". Metta Kicnau-Verlog, Liineliurg: 1951 .

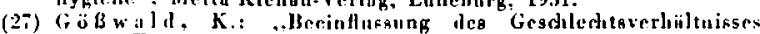
lured Tenperatureinwirkung bei Formica rufa $\mathrm{J} . "$ in ,. Die Ynturwise. $42,13.3 .7 .74 .195 .5$.

(26) $G$ ö $\beta$ wald, K.: "Obersitht äber die vom Institut fiir Angu watedte Zoulogie der Univerritü Würburg durdegefülirten Ver mehrungen der Hotes Waldameise" in "Waldhygirae", ], 7"-82. 195 i.

(29) I к \& 1. B. ". W. , Yersudse zur Ansiedlung von Waldfleder mïuren" in Filedlermonsküsten" in "Forstwirtedi. Centralbl.", $\rightarrow$ $193-210.195 .5$

(30) K a r f iat, H.: .. Yeue Wege der Populntionsanalyse an rindenbewohnenden Arthropoden" in ... Nardiriditeubl. Deutsd. I'llanzenshituts. “. Brawlindeweig, $\overline{7}, 133-136,1955$.

(31) Klrin.Kra u theim, F.: ..Zur Obologic des Kartoffelküfrs.

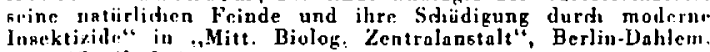

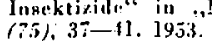

(32) Kioft. W: . Waldameisen und Pfanzenlänse“ in ,Allg. Forst. rritstir." $8,520,1953$

(33) Kripg, A.: "Tntersuduozen iber die Polycilrose von Niso diprion sertifer (Gcoffe.)" In "Ardh. f. d. ges. Virusforsdumb" 6. 16.3-17., 1955.

(31) Krieg, A.: ..Lithe und elektronennikroskopiodie Linterbuchun. gen zur Patholugie der .Lorsder. Erkrankung" von Engerliafer und zur Zytolopic di:r Nidietsia melolonthac nov. opec." in .Zvitatir. Niturfored, ", $106,31-37,1955$.

(i5) Kriet, A.: ,Cher Inlektionskrankheiten hei Engerling"n wn Melolontha spec. unter besonderer Beriidsidntigung einer Mioro. sporidien.Erkrankung" in ..Zentralbl. f. Bakt., Parasitenkile. laf.ktinnsknnkh. ". Hygiene". 11. Abt., 108, 535--538, 1955.

(36) Krieg, A.: ,Zur Differenzicrung von Virus.Krankheiten bei Intekten" in .Mikrogkopie". W'irn, 42,609-610. 195.3.

(37) Krieg, K.: ..Interkudungen zur Wirbeltier-Pothogenitut und

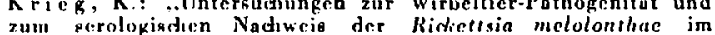

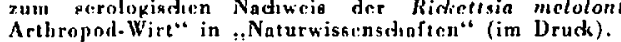


(3ii) K, i g , A.: ", Eodogeue Viruyentstelung" utul Lotenzproblem

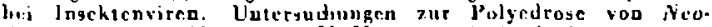
dijerion sersifer (Geoffr.)"., II. Mitt. in , Ardi. f. dl. ges. Virus. forkitig." (im Druek).

(34) Ku bu, K.: (Bricflidıc Mitteilung vom 2. 10. 1951).

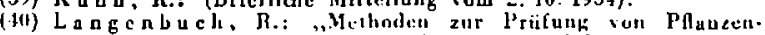

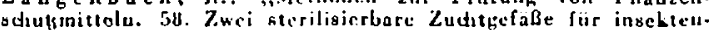

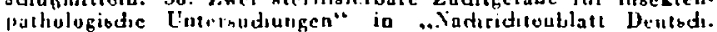

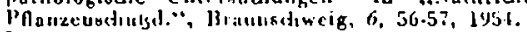

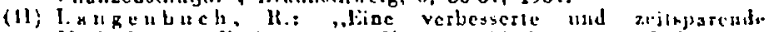

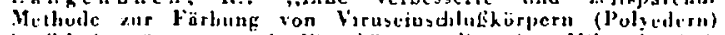

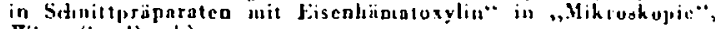
IIin: (im Druts)

(12) Lril, E. u. Uls chowy, G.: ,I,uthdethafteprlege und laud.

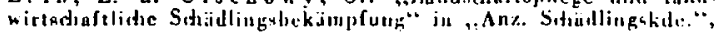
2A, $115-150,19.55$

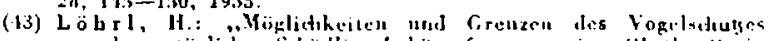



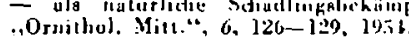

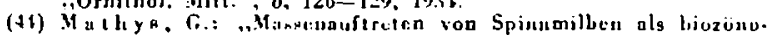

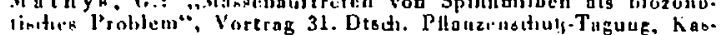

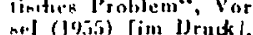

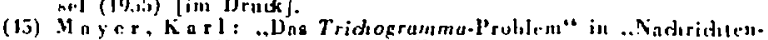

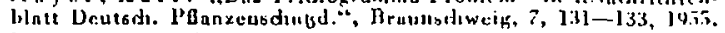

(16) Miller, E.: ..Konnen Honigton liefermele Bnumliinse (Lachni.

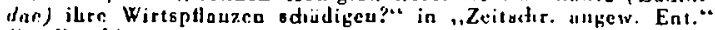
(iin Druds)

(17) Müller-Kögler, E.: ,Die Jurstirr Krankheil" der Maj. kufurengerlinge" "ll ,Allg. Furmlatitndar.", 9, 457-4.i8, lyst.

(18) NikJas, O. F.: "Untersudungeu uiber la Auftreten con Krankheiten uod Sdï̈ligungen, insbesoudore über Jio ,Lorbcter Selutie (Ridiedesia meloloniha Krieg) in Frrilundpupulitionen des Muikiler-Fongerlings (Mcloluntha opec.)" in ,Zeitstir. Ptlan.

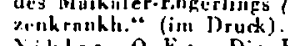

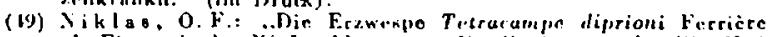

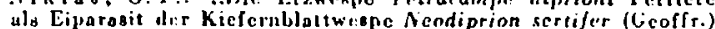

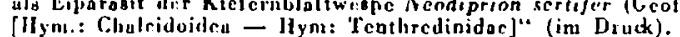

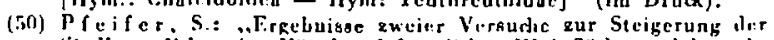
Siedlungudidite der Vügul anf forstlidicr Kleindüdie und lienuthloarter Croßnäilie" in ,Wuldliyginac", 1, 76-78, 19.55 .

(51) Pfeifer, S.: ..Experimentelle Untersudiuagen uad Frcilant. lieobiatitungen zur Fegtotellung ier vertilgung dea kartuffelkiifern (Joptinotarsa decemlinnata Say.) durch mitteleurupuibde Vogelnten" in ,Zcitidir. angew. Ent." "37, 447-161, 195..

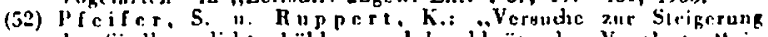
ler Sicdlunguditite liöhlea. und busdibrütemiler Vogrlarten" in .Biol. Abliandl." Heft 6, 1953.

(5i) ïickett, A. D. u. Pat tersun, Y. A.: "The influence of Epray prokrame on the fauna of apple ordiards in Nova Scotin. IV. A review" in "Conad. Eutom.", 85, 472-478, 1953.

(i.t) $O$ und "u u, W.: . Uber cinige neue Trichogramua-Wirte und ihro Stellung int Wirt-l'arasit.Verbälnio. Ein Beitrag zur Ana. lyye des Poragitismus bei Sthlupfwespea" in ...Padirichtorublate

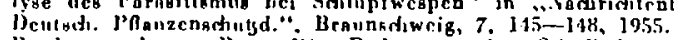

(.5) Rademecher, B.: „Dic Bedeutung der Sdaflialiung in

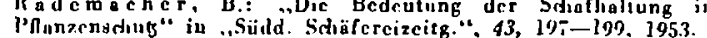

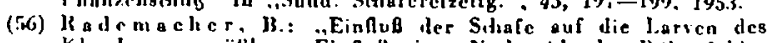
KJece-Luzernenrüblera. Eindub eincr Nachwcide der Hühenfelder nit Sctiafen euf den Bifall der Rüben mit Cercospora. Hlattfledk.... krankbrit." (linverïffentlictiter Beridit.)

(5i) Scherney, F.: ., Linterkudiungen ïlocr Vorkommrn un! wirt.

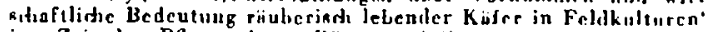

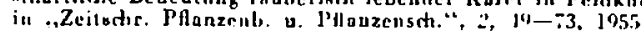

(50) Schituitachok, E.: .Fragen sler Waldhygienc" iu ..Angen l'flouzeusuziclogic." Verüf. d. Kiirmtacr Landesinst. f. angew l'fonzensodiulogie, Klngenfurt, Feststirift Aidtioger, 2, 1011 bis l11:2ll, 1105.4 .

(59) Schlabritzky, E.: „Dus Sultearter Inocklarium zur Zutu

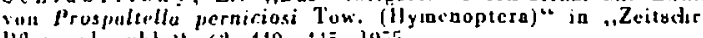

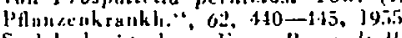

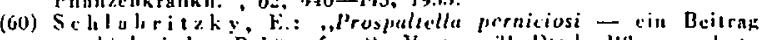

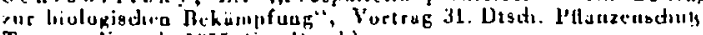

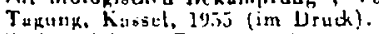

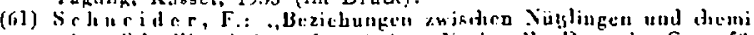

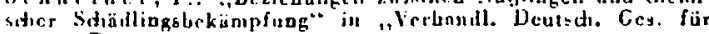
ing1.. E. E

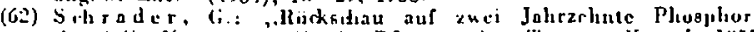

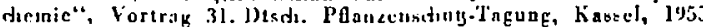
(iin l)rudk).

(63) Srlü̈te, F.: ..Lateroudiungen über die l'opulntioasdyumik

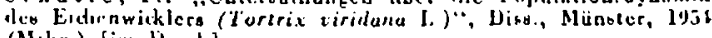
$\left(\mathrm{M} ; \mathrm{kr}\right.$.) [im $\left.b_{\mathrm{r} u \mathrm{k}}\right]$.

(6.1) Sehwerdifefer, F.: ,Grundoiblidice zur Inpulation,

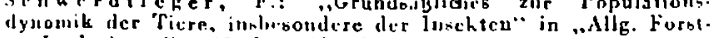
dy"lomk der ricre, inhlwbondere der



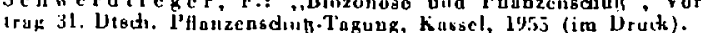

(bi) Schnitulla, H.: ,Lotrsudiungen zur biologigdiea Bc

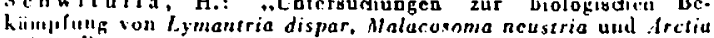
reja" Dibuertation, Zool. Inst. Köla, 19j5.

(6i) Solonou, M. E.; .Das Gleidigewidst ion Iubekteulsesilkerunger und dic diconisdic Sdiädlingsbekürofifung. Sdiädlinguver mchrumb "1s Folke vou lusektizidbeliundlumg" in ..Zeitsdir. "lngew. Ent.", 37, 110-121, 1955. (Uberackung der engl. Original. "ribcit: "Insciet population baluece and deaniral control of perate. l'ret nuthrenhe induced loy sprenyiles" in "Clecmistry und In duetey". 1143-1147, 1953. Cbererigt von J. F r a nz.)

(68) Steiace, H.: .Uber ien Einflub dirmiudier Mittel unf die

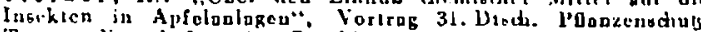
Tagung, Kassul, 1955 (im Druck).

(6!) Steing a 6 . E. A.: "The cffects of diseage un insect popu. lations" in "Ililgardia", 2.3, 397-261, 19.54.

(i0) Thaleuhorot. W.: "Probleme der biologisdien Bekümpefung

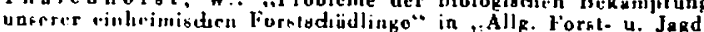
z'ituog". 125, 2:1-227, 3254.

(7) Thiel m n u, K.: .Zur Problematik d.r neutron Entwicklung lici Jer Furatsdiüllingsbekänplung" in "Allg. Forbtzituder." III. $470-171,1955$.

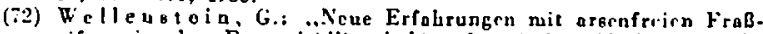
giftill iu der Forstediadlingubekämpfung" in "Verl. Deuted.

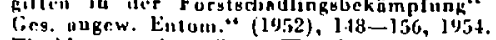

(3) Wellenstein, G.: "Wag kinnen wir voll der Roten Walde

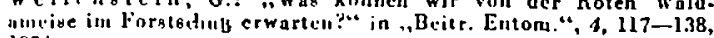

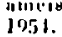

(i) N' prohlemm" in ..Mitt. Hinlog. Hundesanstali f. IAnd- u. Foret virtart..", (83), $17--37$. 1955.

(i5) Yullirr. M. A.: "An unnotated bihliogrophy on Aphrlinus mali (Hald.), "parasito of the woolly apple ophide" in "U.S. Dep. Agric.", Bureau Entum. P'lant Quorantiue, E.B61, 1953.

(76) Zncloclei , G.: ,Zur Finwirkung never locrhtenkampfutuffe

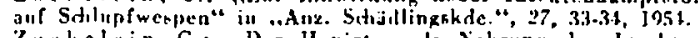

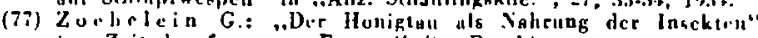
in ..7.eithtir. f. angew. Entun." (isn Dende).

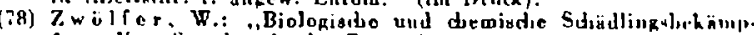

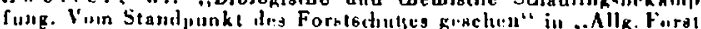
zritung $"$, 8. $.19-3.5$, 19.3.7.

Pflanzensduyamt des Landes Schlesteig-Holstein, Bezirksseelle für Pflanzenschü Rellingen

\title{
Ein Beitrag zur Frage der Behandlung blühender Pflanzenbestände mit Insektixiden
}

\author{
Von Dipl.Biol. H. FABCR
}

Boti der intensiven Bewirtsdiaftune unserer KulturAiidien wird es oft erforderlich, zur Vernidntung schüdlicher Insektcn Pflanzenbestände anch zur Zrit der Blïte mit insektrnabtötenden Miltch zu bohindeln. Dic meisten unserer Kulırplanzen sind aclbststeril und uur wenige sclbstfertil. Sic sind unf I'remdlestäubung angowiesen, dic - sowirit nicht Windbestiubung in Frage: kommt von Insekion durctigeführt wird. Um also die Erträge zu sidırı, müssen die nüglichen Insckten als Pollenüberträger gescliont, dic schiidlidien Insckten als Ertragsminderer vernidhtet werden. Hicr rrgibt sid, eine Diskrepanz, die cine gonaue Prüfung der Ferhibluisso erforderlich madit, um zu entacheiden, ol, eine Bchandlung blähender Pflunzen mit inscktenabtöteuden Mitteln vertrelbar ist.

Der widıtigste Bestäuber unserer Kulturpflamzen ist die Honigliene. Die kurze J3lïtezeit in dent unsgedehnten Monokulturen erfordert für die Pollenübcrtragung einc grobe Anzahl von lnsekten. Dicse stcht uns im Biencuvolk mit scincn uusähligen Arbeiterinmen zur Verfügung. Bcim Besuch der Blüte bleiht Pollen an den feinen Borsten deb Kopfes und Körpars der Bicne hängen, der heim Herumkricchen der Biene in ler nächsten Blüte mit der Narhe in Berülirung koment und abgestreift wird. So wird ein mn. freiwilliger Pollentranspurt und danit dic Befruchtung durdigefïhrt.

Aus der Erkenntuis heraus, duB жur Erhöhmo der Erträge dic Anwesenhojt von Honigbienen unbedingt orforderlid ist, werden jährlide z. B. zur Zcit der Obstloaumund Rapsblizte 'Fausende von Bienenvölkeru in die baupt. sächlidisten Anhaugchiete geholt. In cinigen Golietrn werden sogar für die Einwanderung Geldpränicn an die Imker grezuhlt. Um nun diese nertvollen Blülenbestaiuber vor der Vernichlung durct unsadigemäße Anwendung vou Inscklizidin zu bewuhreu, wurde durdh die Virorduung 